Bull. Mater. Sci., Vol. 4, Number 3, May 1982, pp. 193-206. (C) Printed in India.

\title{
Vapour phase crystal growth under microgravity environment
}

\author{
VIKRAM KUMAR \\ Department of Physics, Indian Institute of Science, Bangalore 560012, India
}

MS received 27 September 1979

\begin{abstract}
Vapour phase crystal growth experiments performed in the Skylab and ASTP missions are reviewed. The basic vapour phase crystal growth technique is described and effect of gravity is discussed. The multipurpose furnace specially designed to carry out various experiments in flight conditions is described. Ge Se, $\mathrm{Ge} \mathrm{Te}$ and $\mathrm{GeS}$ as well as ternary $\mathrm{GeSe}_{0.89} \mathrm{Te}_{0.02}$ and $\mathrm{GeS}_{0.98} \mathrm{Se}_{0.02}$ crystals have been grown in space showing improvement over similarly grown crystals on ground as determined by $x$-ray diffraction, chomical homogeneity and surface morphology studies. Mass ftux rates under microgravity conditions have been found to be up to 10 times larger than expected indicating need for better theoretical and experimental understanding of the effect of gravity on crystal growth.
\end{abstract}

Keywords. Crystal growth ; microgravity onvironment; space.

\section{Introduction}

One of the most impartant techniques used for growing bulk or layer-type single crystals is chemical vapour transport (CVT). Materials used for electronic device such as elemental and compound semiconductors can be grown by this process. This technique also permits crystal growth of relatively non-volatile materials at temperatures well below their melting points. In this method the species to be deposited are transported from a source to the seed by chemical reaction at the source, transport by an agent across a temperature gradient, dissociation and deposition at the seed. Generally bulk crystals are grown in closed tube and layertype structures in open tube systems.

The performance of electronic devices and materials is known to be critically dependent on chemical homogeneity and crystalline perfection. Semiconductor industry requires large and perfect crystals. These requirements can be satisfied in ground-based systems only to a limited extent due to the presence of gravity. In such systems, the gravity-driven convection interferes with the mass-transport and the deposition processes disturbing therefore the homogeneity, the perfection and consequently the performance of the material.

Vapour phase crystal growth in space is expected to yield better crystals due to reduced gravity-driven convection. It is expected also to lead to fundamental data and help in understanding transport and growth processes.

The purpose of this paper is to review the vapour phase crystal growth experiments performed in the Skylab and the Appollo Soyuz Test Project (ASTP). First 
the vapour phase growth technique and theory will be discussed in brief. Next, the furnace requirements in space and how they were dealt within the ASTP mission will be described. Then the experimental results will be reviewed and finally some of the future proposals will be discussed.

\section{Basic technique on ground}

The technique under consideration, requires a reversible chemical reaction between a solid and a gas resulting in one or more gaseous products. For example

$$
\mathrm{GeSe}(\mathrm{s})+\mathrm{GeI}_{4}(\mathrm{~g}) \rightleftharpoons \mathrm{GeI}_{2}(\mathrm{~g})+0.5 \mathrm{Se}_{2}(\mathrm{~g}) \text {. }
$$

The forward reaction takes place in the source region of the reaction vessel. The vapour species migrate to another region where the reverse reaction takes place. Well-defined crystals of the solid source can be abtained by condensation under proper conditions. Thus the salid is transported via the gas phase by means of a chemical reaction (Laudise, 1970).

The migration of gaseous species is generally achieved by a temperature difference. The transport can occur from high to low temperature or vice versa depending on thermadynamic properties of the reaction.

While the chemical reactions can be assumed to be independent of gravity, the main difference between the earth and space based vapour phase crystal growth comes in the mode of transport.

There are essentially two different mechanisms for transport in vapour phase:

(i) Diffusion accurs whenever a concentration gradient is present.

(ii) Convection arises due to the interaction between the gravity and density differences caused by the temperature gradient.

Generally these transport mechanisms occur simultaneously. At very low pressures $\left(10^{-3} \mathrm{~atm}\right)$ the transport rate is controlled by chemical reactions at the source and seed zones. At medium pressures ( $\leqslant 1 \mathrm{~atm})$, the dominant mechanism is diffusion and the diffusion coefficient is inversely proportional to pressure. At higher pressures $(>1 \mathrm{~atm})$ thermal convection predominance increases with increasing pressure.

Wiedemeier et al (1975) carried out detailed studies of transport rates of GeSe, $\mathrm{GeTe}$ with $\mathrm{GeI}_{4}$ and $\mathrm{GeS}$ with $\mathrm{GeCl}_{4}$ as transport agent. The flux is calculated from the amount of crystals recovered, dimensions of the tube and the duration of the experiment. Their results for ground based studies are shown in figure 1. Here we clearly see the diffusion-controlled and the convection-controlled regimes of transport. Additionally, they have observed a decrease in crystal perfection with increasing convective component in the overall transport process.

\section{Apparatus}

The crystal growth and other materials process ng experiments in the Skylab and ASTP missions ware performed in a multipurpose furnace (figure 2) designed by Boese et al (1977) of Westinghouse Carporation to provide wide predetermined temperature-time profiles. The improved ASTP version of the furnace consisted of 


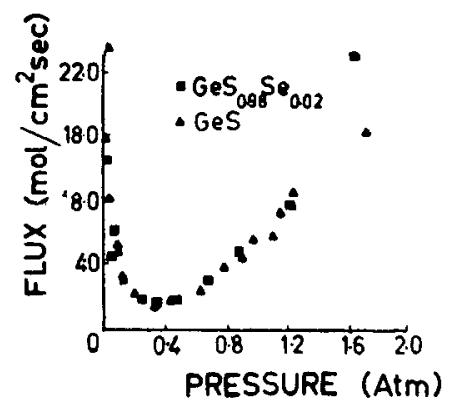

Figure 1. Mass transport rates in torms of flux as a function of transport-agent pressure for the temperature gradient $677-788 \mathrm{~K}\left(604-507^{\circ} \mathrm{C}\right)$ determined under ground-based conditions.

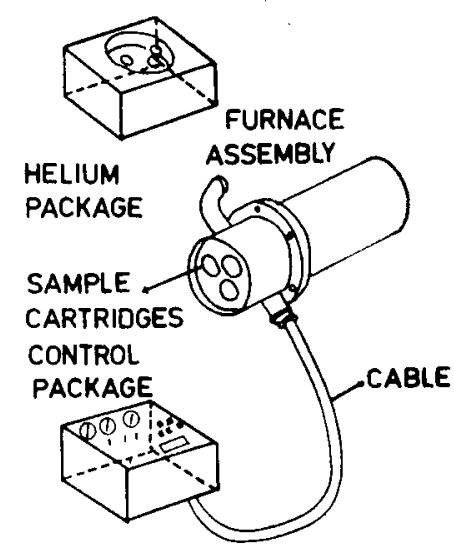

Figure 2. Multipurpose electric furnace system.

three parts (figure 3): the furnace, the controller and the He rapid cooldown system. The system was designed to attain $1150^{\circ} \mathrm{C}$ with $205 \mathrm{~W}$ of available power.

The resistance-heated furnace itself contained three tubular cavities for metal cartridges and ampoules for various experiments. The total length of about $12.7 \mathrm{~cm}$ could be divided in three parts (figure 4 ). The heat leveller region was surrounded by graphite and provided axial and radial temperature uniformity of less than $1^{\circ} \mathrm{C}$. This region was heated resistively and a great deal of meticulous considerations (figure 5) went into its final design. The gradient region was a stainless steel tube. The heat extractor region was surrounded by copper. Since the furnace was heated only in the heat leveller zone, the desired temperature gradients and stability could be achieved by introducing heat shields around the quartz ampoules within the metal cartridges.

The entire furnace was operated under vacuum which was readily achieved by connecting a line from furnace through the docking module into the space.

The helium rapid cooldown system (figure 6) was necessary to reduce the turnoff to touch time from $20 \mathrm{hr}$ to $3 \mathrm{hr}$. Ordinarily the furnace was operated in vacuum. Introduction of helium enhanced the thermal transfer process. It was 
found that only $0.01 \mathrm{~atm}$ pressure is sufficient to achieve maximum efficiency and for this only $0.16 \mathrm{cc} \mathrm{He}$ under standard conditions was enough. The system consisted of a helium storage tube, a dosage cavity and three valves along with associated lines. Helium could be introduced into the furnace by opening valves $B$ and A sequentially to achieve rapid cooldown. After cooldown and before opening, the furnace could be brought to docking module atmosphere by opening valves $A$ and $C$ simultaneously.

A chramel-alumel thermacauple was used to monitor the temperature of the heat leveller region. The contral instrumentation provided proportional control with variable controlled cooldown rate and shorted thermocouple protection.

\section{Experiments in space}

The group led by Weidemeier (1976) has carried out vapour phase growth experiments in bath Skylab and ASTP missions. The materials grown were GeSe, $\mathrm{GeTe}$ and $\mathrm{GeS}$ as well as their ternary mixtures to test the chemical homogeneity. These were prepared from high purity elements $(10 \mathrm{ppm})$. High purity $\mathrm{GeI}_{4}$ and $\mathrm{GeCl}_{4}(10 \mathrm{ppm})$ were used as transport agents. These materials were enclosed in a quartz ampoule under $10^{-6}$ torr pressure. The growth conditions for various experiments are listed in table 1.

Table 1. Experimental systems and mass transport rates

\begin{tabular}{|c|c|c|c|c|c|}
\hline $\begin{array}{l}\text { System } \\
\text { material }\end{array}$ & Carrier & $P$ (atm.) & $\begin{array}{c}\text { Mass flux } \\
1 \mathrm{~g}\end{array}$ & $\begin{array}{l}1 / \mathrm{cm}^{2} \sec \\
\text { micro-g }\end{array}$ & $\begin{array}{l}\left.10^{-0}\right) \\
\text { expected } \\
\text { diff. mode }\end{array}$ \\
\hline \multicolumn{6}{|c|}{ Skylab $3\left(524-422^{\circ} \mathrm{C}\right)$} \\
\hline GeSe & $\mathrm{GeI}_{4}$ & $1 \cdot 5$ & 39 & 5 & 0.5 \\
\hline GeSe & $\mathrm{GeI}_{4}$ & 0.13 & $5 \cdot 3$ & 10 & $3 \cdot 5$ \\
\hline GeTe & $\mathrm{GeI}_{4}$ & 0.75 & $2 \cdot 5$ & 3 & 0.5 \\
\hline \multicolumn{6}{|c|}{ Skylab $4\left(412-346^{\circ} \mathrm{C}\right)$} \\
\hline GeSe & $\mathrm{GeI}_{4}$ & 0.42 & 0.9 & 0.8 & 0.15 \\
\hline GeSe & $\mathrm{GeI}_{4}$ & $0 \cdot 12$ & 0.8 & $1 \cdot 8$ & $0 \cdot 35$ \\
\hline GeTe & $\mathrm{GeI}_{4}$ & $0 \cdot 33$ & $1 \cdot 7$ & $1 \cdot 0$ & $0 \cdot 40$ \\
\hline \multicolumn{6}{|c|}{ ASTP $\left(604-507^{\circ} \mathrm{C}\right)$} \\
\hline $\begin{array}{l}\mathrm{GeSe}_{0.99} \\
\mathrm{Te}_{0.02}\end{array}$ & Gel, & 0.7 & 7.9 & $4 \cdot 2$ & $1 \cdot 4$ \\
\hline $\begin{array}{l}\mathrm{GeS}_{0 \cdot 98} \\
\mathrm{Se}_{0.02}\end{array}$ & $\mathrm{GeCl}_{4}$ & 0.96 & $22 \cdot 1$ & $22 \cdot 1$ & 6.5 \\
\hline $\mathrm{GeS}+\mathrm{Ar}$ & $\mathrm{GeCl}_{4}$ & 0.96 & $15 \cdot 0$ & $13 \cdot 0$ & $2 \cdot 8$ \\
\hline \multicolumn{6}{|c|}{$+P(A r)=1.91$} \\
\hline
\end{tabular}




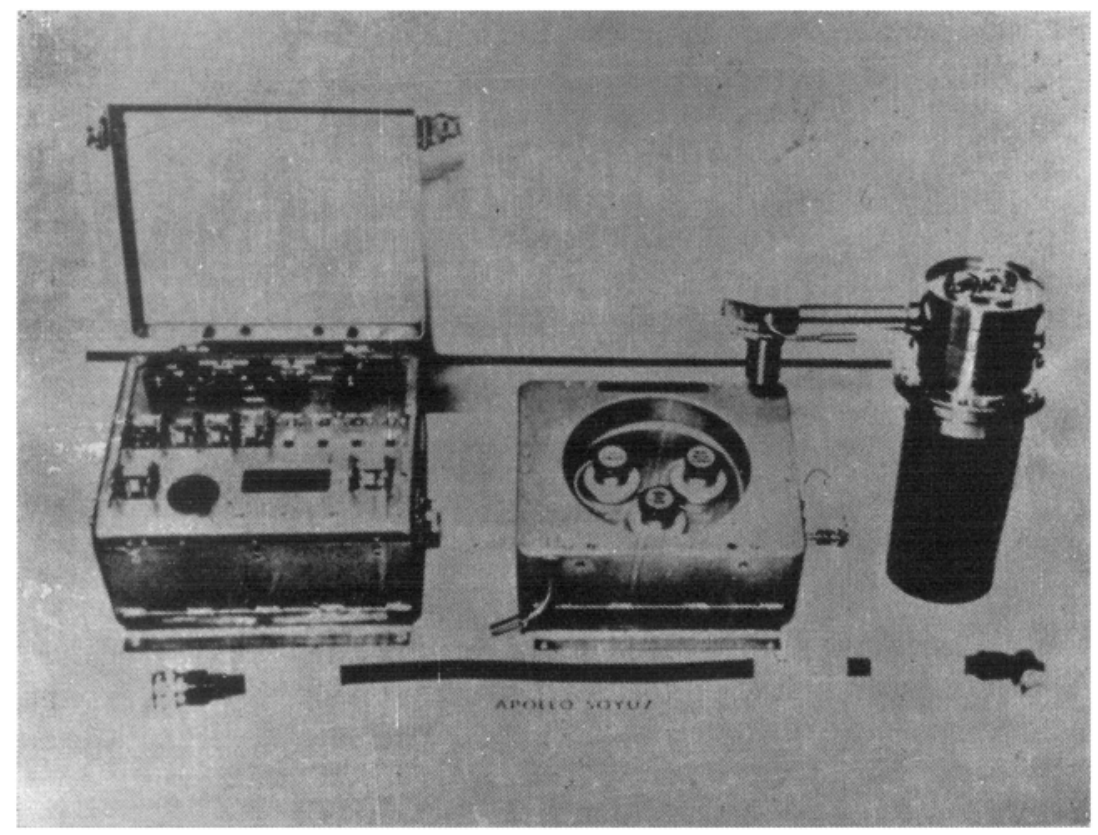

Figure 3. The ASTP multipurpose electric furnace system. 



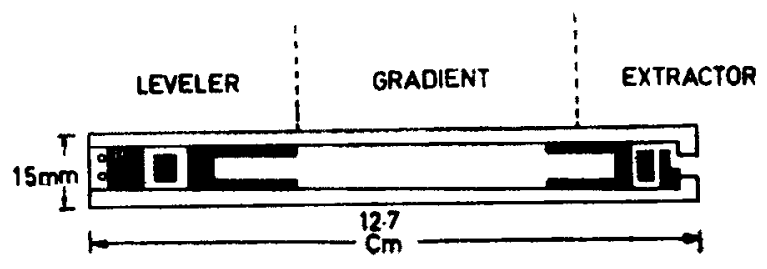

Figure 4. Ampoule design of the multipurpose furnace.

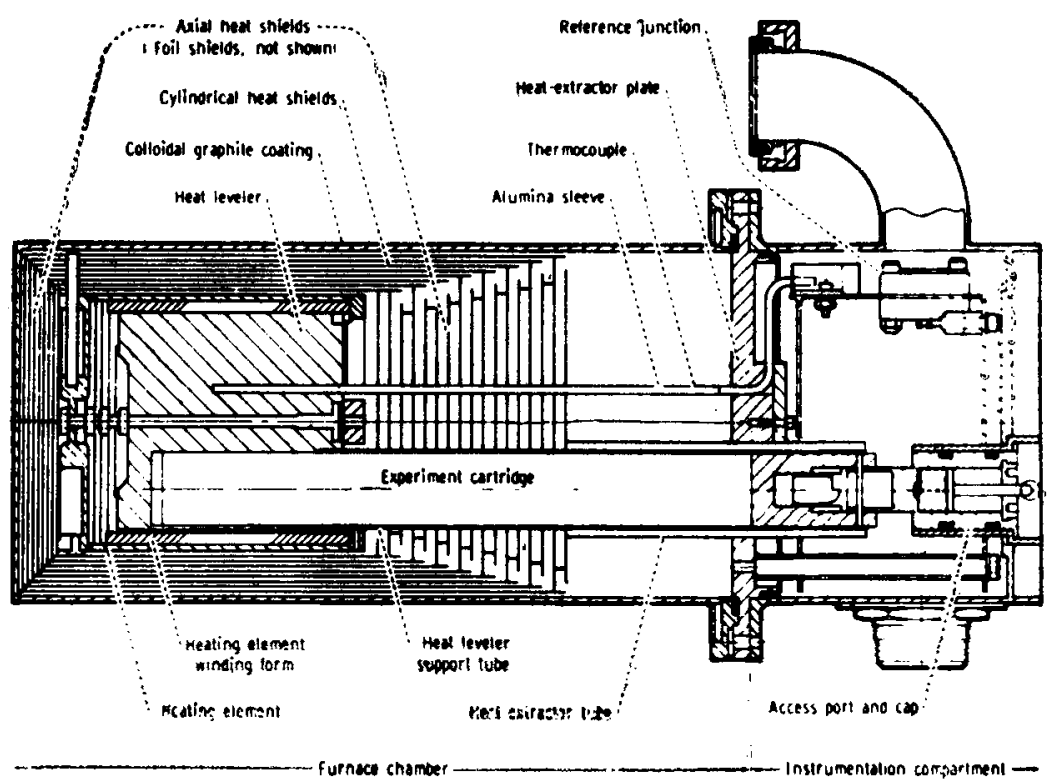

Figure 5. Shamatio of tho miltipurpose furnace used for the ASTP crystal growth and solidification experiments.

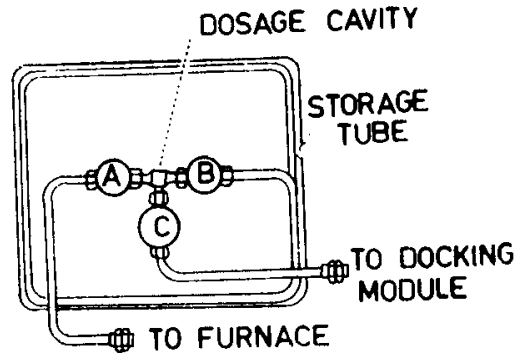

Figure 6. Sohematic representation of the helium rapid cooldown system.

The ampoules were enclosed in hermetically sealed metal cartridges which were then inserted into the multipurpose furnace by the astronauts. The temperature time sequence for ASTP mission is shown in figure 7. Identical experiments were carried out on ground for comparison. 


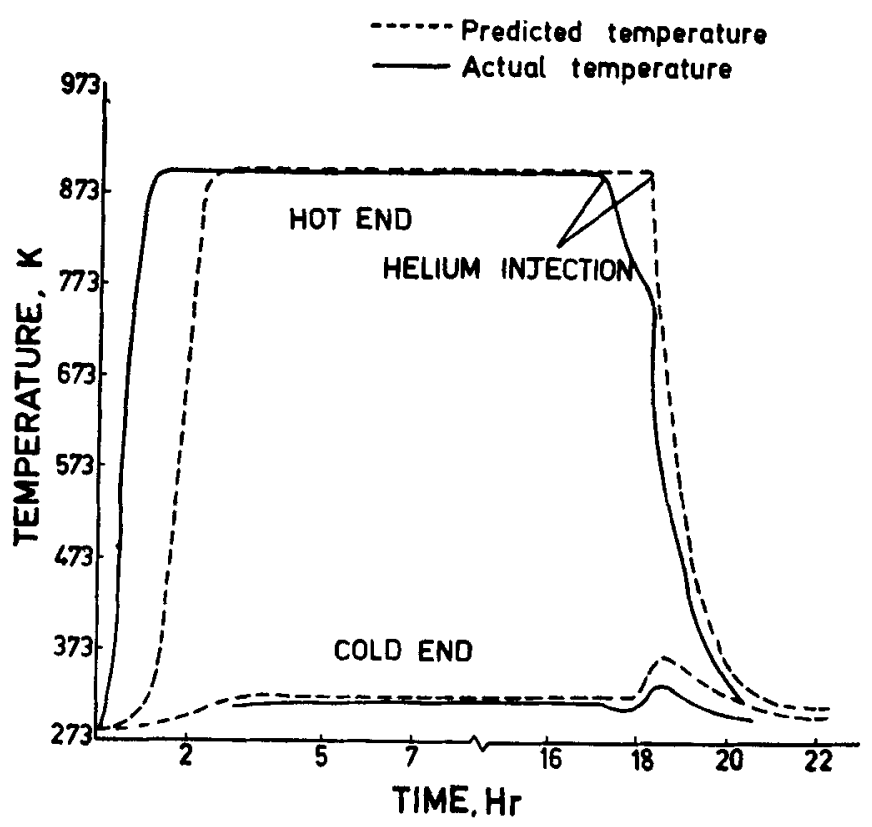

Figure 7. Temperature profile for crystal growth from the vapour phase.

\subsection{X-ray studies}

First the crystallographic structure of the crystals was studied using $x$-ray powder and Laue diffraction techniques, using $\mathrm{CuK}_{a}$. radiation and conventional instruments. The data for space and ground grown crystals showed excellent agreement. The orientation of the predominant faces of single crystals was found to be (001). The lattice constants $a, b$ and $c$ also showed no effect of gravity within the experimental detection limits.

The Laue photographs, though indicating some orientation, show considerable differences between space and ground grown crystals. The upper half of (figure 8) shows Laue patterns for $\mathrm{GeSe}_{0 \cdot 99} \mathrm{Te}_{0 \cdot 01}$. The ground-grown crystal on the left shows striations and multiplicity of diffraction spots. The Laue pattern of the space-grown crystal has much better definition of spats. This indicates less strain and inhomogeneities and improved crystallographic perfection of the space-grown crystal over the ground-grown sample.

The Laue patterns of $\mathrm{GeS}_{0 \cdot 98} \mathrm{Se}_{0 \cdot 02}$ similarly show considerable improvement in crystallographic perfection of the space grown crystal as shown in the lower half of figure 8 .

\subsection{Chemical homogeneity}

The ASTP experiment was specifically designed to study the effect of gravity on chemical homogeneity. For this purpose mixed crystals $\mathrm{GeSe}_{0 \cdot 09} \mathrm{Te}_{0_{0} \cdot 01}$ and $\mathrm{GeS}_{0.98} \mathrm{Se}_{0 \cdot 02}$ were grown. The distributions of trace elements Te in GeSe and $\mathrm{Se}$ in GeS were measured using electron microprobe analysis. Their concentrations were measured along $a$ and $c$ axes over several points. It was found 

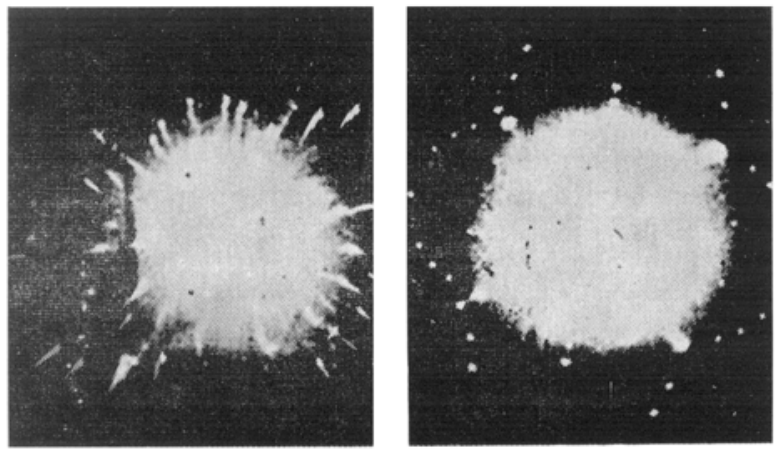

(8)


Figure 8. I La in jattern for $\mathrm{GoSc}_{0.99} \mathrm{Tc}_{0 \cdot 01}$ (upper) and $\mathrm{GeS}_{0.98} \mathrm{Te}_{0.02}$ (lower) grown under ground (left) and spaco (right) conditions. 




a

(9)

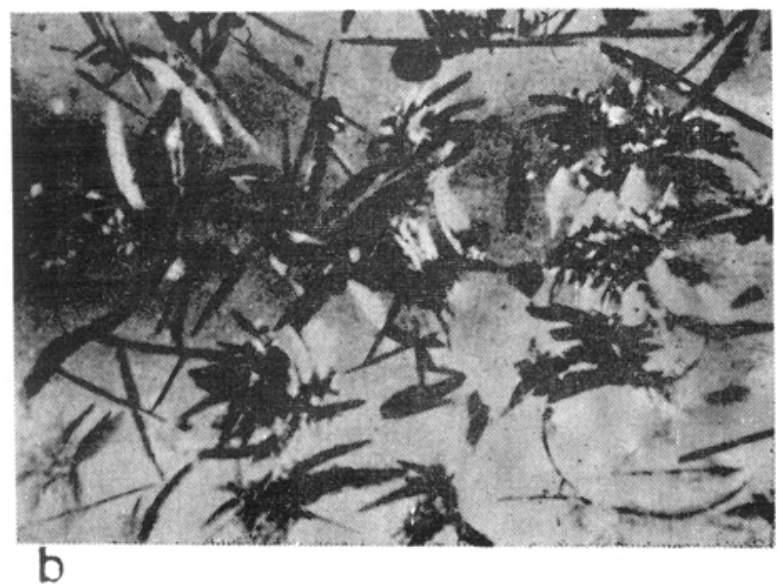

Figure 9. Deposition pattern of transport agent $\mathrm{GeI}_{4}$ on ampoule walls: (a) in microgravity environment, (b) under ground-based conditions. 




Figure 10. Roprcsintative crystals of $\mathrm{GeS}_{0.08} \mathrm{Se}_{0.02}$ : (a) ground-based, (b) spacegrown. 

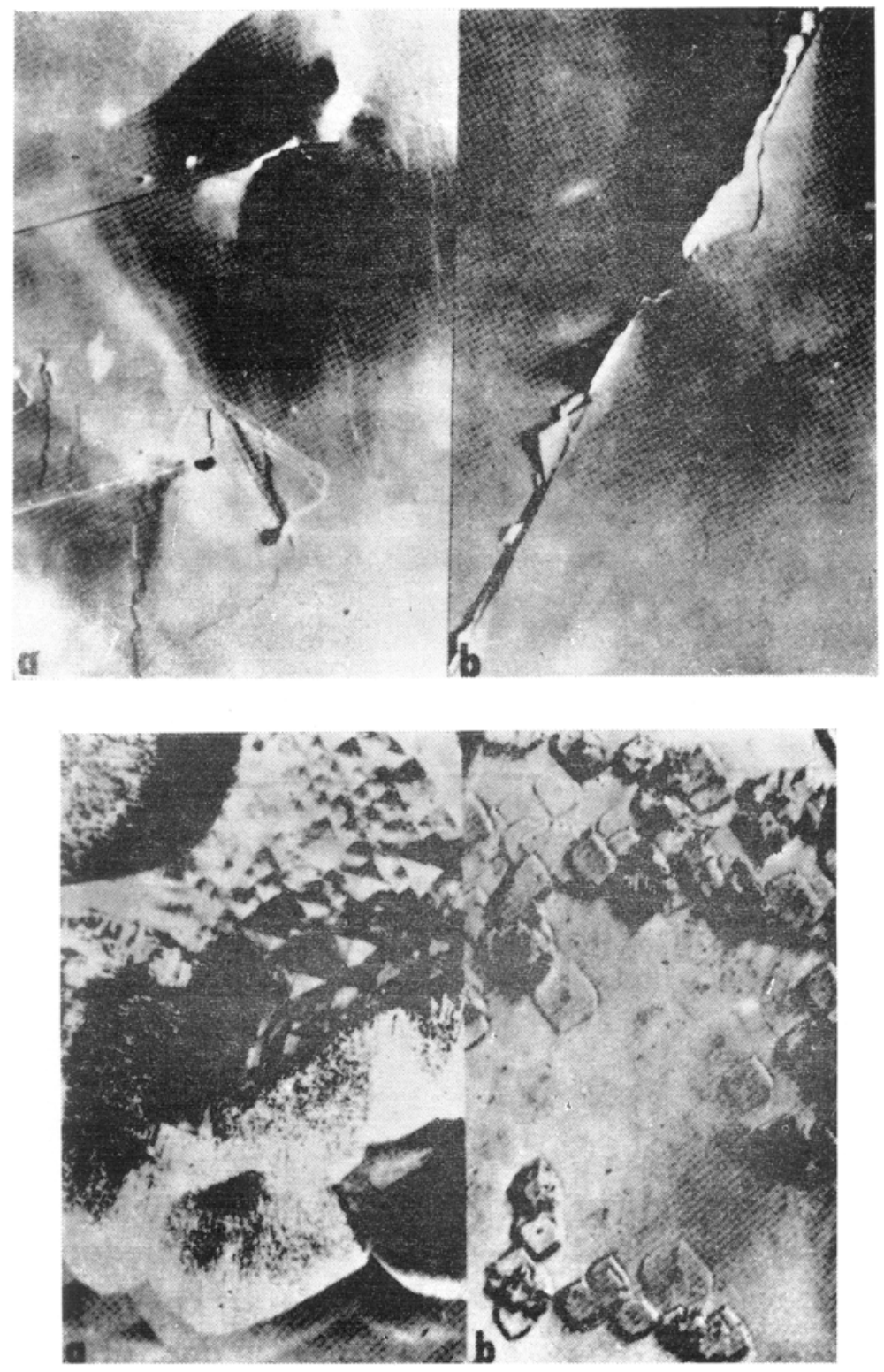

Figure 11. Scanting electron photographs $(4.00 \times)$ of cleaved $(001)$ faces of GeSe (a) ground-based and (b) spacc-crystals.

Figure 12. Optical photomicrographs $(125 \times)$ of cleavod (001) faces of GeSe : (a) ground-bascd and (b) spaca-crystals both after thermal etching $\left(2.5 \mathrm{hr}, 200^{\circ} \mathrm{C}\right)$ 
that the space-grown orystals were much more homogenous than the ground-grown samples. For example $\mathrm{Te}$ in GeSe was found to be $1.71 \pm 0.6$ in ground grown sample and $1.52 \pm 0.08$ in the space-grown sample along the $a$-axis.

\subsection{Morphology}

The crystals were examined under optical microscope and scanning electron microscope. They were also subjected to cleaning and etching to study defects.

First of all, the crystallites of the transport agent $\mathrm{GeI}_{4}$ deposited on the ampoule walls were examined under microscope (figure 9). Under microgravity, $\mathrm{GeI}_{4}$ is deposited in the form of well-developed, transparent single crystal platelets and needles. The corresponding ground based experiment resulted in $\mathrm{GeI}_{4}$ deposition in dendritic-type growth with a distinct curvature. The absence of any distinct curvature in the space ampoule reflects a more homogenous gas motion in microgravity environment.

Photomicrographs of representative $\mathrm{GeS}_{0 \cdot 88} \mathrm{Se}_{0 \cdot 02}$ crystals grown on ground and in space are shown in figure 10. Comparable results were obtained for all systems grown in Skylab and ASTP missions. Generally the ground-grown crys tals show rugged edges, intergrowth, stepped and rough surfaces. The spacegrown crystals revealed definite improvement in terms of surfaces and edges.

Generally the SEM photographs of corresponding space and ground-grown crystals show more inhomogeneity in ground-grown crystals. For example, as shown in figure 11 the cleaved face of GeSe, grown in Skylab mission, is much more regular and the cleavage separating (001) planes of different depth from the native surface is clearly seen. The ground-grown crystal shows much more irregularity.

Similarly a study of etch pits on cleaved planes shows vast improvement generally in space-grown crystals. For example, figure 12 shows cleaved and etched faces of GeSe crystals from Skylab mission. The ground-grown crystals yield an etch pit density in upper $10^{5} \mathrm{~cm}^{-2}$ range with pits as large as $10^{-2} \mathrm{~cm}$. The etch pit density of the space-grown crystals is in the range of $10^{4} \mathrm{~cm}^{2}$ with largest pits about $10^{-3} \mathrm{~cm}$.

The combined results of morphological studies on crystals grown on earth and in Skylab and ASTP missions demonstrate considerable and definite improvement in space grown crystals.

\subsection{Mass transport rates}

Mass transport rates were determined from the mass of recovered crystals, the geometry of the ampoule and the duration of the experiment. Uncertainties in the amounts of recovered crystals were combined to yield lower flux values. The observed flux values for both the Skylab and the ASTP experiments are listed in Columns 4 and 5 of Table 1 for ground-based and space crystals respectively. The expected diffusion mode mass flux values in column 6 are obtained from the extrapolation of the diffusion branch of experimental flux rates obtained in the ground-based studies.

Rather unexpected flux values have been observed. Generally the microgravity flux rates are 3-10 times larger than the expected rates. Further, in some cases, 
the flux values for space are actually greater than the observed values on ground despite the expected suppression of convective mode of transport. The ratio of microgravity to ground-based fluxes generally decreases with increasing pressure with a value $>1$ for the lowest pressure.

The observed unexpected transport rates in space have remained unexplained and further theoretical and experimental studies are necessary to resolve this anomaly.

\section{Conclusions}

Thus the vapour phase crystal growth experiments conducted so far have measured upto the expectations. They have demonstrated clearly that the space-grown crystals are generally more perfect than the ground-grown crystals in otherwise identical conditions. They have also exposed the need for better models of mass transport, thus leading to fundamental understanding of the effects of gravity.

For the forthcoming Spacelab, scheduled for 1980 several vapour phase crysta growth experiments have been proposed, Belouet $(1976,1979)$ and Wiedemeier et al (1980) have proposed to further carry on their work with CVT growth of GeSe and related compounds, Laynay has proposed a chemical vapour deposition (CVD) growth of vanadium oxide which has a sharp metal-insulator transition, presumably highly sensitive to lattice disorder. Belouet (1979) has proposed growth of $\mathrm{HgI}_{2}$ using the evaporation-condensation process hoping to get more fundamental data on evaparation and diffusion processes.

In conclusion, the vapour phase crystal growth experiments in microgravity environment provide the crystal growers and the scientists an opportunity to grow perfect crystals and gain additional fundamental knowledge.

\section{References}

Belouet C 1976 ESA Spl Pub No. 114

Belouet C 1979 Thin Solid Films 581

Boese A, McHugh $J$ and Seidensticker 1977 NASA SP 412

Laudise R A 1970 The growth of single crystals (Englewood Cliffis, N.J. : Prentice Hall)

Wiedemeier H, Klassig F C, Irene E A and Wey S J 1975 J. Cryst. Growth 3136

Wiedemeier H, Sadeek H, Klassig F C, Norek M and Santandera R 1976 ESA Spl. Pub. No. 114 